\title{
Evaluation of staging, cytoreduction and second-look operation of 119 ovarian cancer patients
}

\author{
Department of Gynecology and Obstetrics, "Hospital das Clínicas", \\ Ribeirão Preto School of Medicine, São Paulo University (FMRP-USP) - Ribeirão Preto, Brasil
}

\begin{abstract}
Objective:This study was conducted on patients with ovarian cancer in order to evaluate survival. Design: A retrospective study of 119 cases of ovarian cancer from January 1977 to December 1992 with observation until 1993. Location: Department of Gynecology and Obstetrics, Ribeirão Preto School of Medicine, São Paulo University. Participants: Of the 119 cases, 70 (58.8\%) presented epithelial carcinomas and $21(17.6 \%)$ tumors of the sexual girdle/stroma. Data source: The data were obtained from the medical records of the patients. Measurement: Statistical analysis of survival time was based on the nonparametric Mann-Whitney test with the level of significance set at $P<0.05$. Results: The patients with a negative second look had a mean survival of $79.4 \pm 48.5$ months versus $24.2 \pm 15.1$ months for patients with a positive second look $(P<0.02)$. Conclusions: It is concluded that patients with a negative second look present a better prognosis compared to those with residual disease.
\end{abstract}

UNITERMS: Ovarian cancer. Cytoreduction. Second look. Survival.

\section{INTRODUCTION}

$\mathrm{E}$ xfoliation is the best known form of dissemination of ovarian cancer. Even in encapsulated tumors there is the presence of malignant cells inside the peritoneal cavity. These cells are distributed throughout the cavity by the respiratory movements of the diaphragm and by the peristaltic activity of the intestine, resulting in a clockwise circulatory movement of the peritoneal fluid that explains the greater involvement of the right diaphragm. Passage through the lymphatic vessels of the diaphragm may permit access to the pleural space and result in malignant pleural effusions ${ }^{1}$. In cases in which no

\section{Address for correspondence:}

Eddie Fernando Candido Murta

Rua Algén Paixão, 170 - Apto. 202

Uberaba/MG - Brasil - CEP 38060-230 microscopic disease is observed outside the ovary, approximately one third of patients present malignant cells in peritoneal cytology ${ }^{2}$.

Indeed, even malignant tumors apparently confined to the ovaries already present occult metastases, mainly due to the lymphatic drainage of the ovaries. About $5 \%$ to $10 \%$ of tumors in an apparently early stage already present metastases in aortic or pelvic lymph nodes. This percentage reaches about $70 \%$ in the advanced disease ${ }^{2,3}$. Hematogenic dissemination is relatively uncommon. Metastases may occur in the liver parenchyma, especially when the disease is extensive, involving the intestine and resulting in the transport of shed cells into the portal circulation. Metastases of the brain or other sites have been described, especially in the final stages of the disease, perhaps due to the current increased survival of these patients $\mathrm{s}^{4,5}$.

Approximately one third of new ovarian cancer cases belong to stages $\mathrm{I}$ and $\mathrm{II}^{6}$. Despite the good prognosis for patients with localized disease, retrospective evaluations show that $50 \%$ of them suffer recurrence and die despite appropriate treatment. It is also possible that the survival 
of patients with localized disease is underestimated, perhaps owing to poor surgical staging ${ }^{3}$.

The correct surgical procedure for the identification of true early ovarian cancer includes: 1: vertical incision of sufficient extension to permit evaluation of the entire abdomen, 2: total hysterectomy accompanied by bilateral salpingo-oophorectomy, 3 : partial infracolic omentectomy, 4: evaluation of the surface of the tumor to determine capsular integrity, 5 : determination of the possible presence of ascitic fluid or collection of a peritoneal wash for cytologic examination, 6: obligatory biopsies of the following high-risk areas: right and left diaphragm, both abdominal gutters, pelvic and para-aortic lymph nodes, peritoneal surface, and biopsy of any suspicious lesion.

After these precepts are observed, the type of subsequent treatment and the patients who no longer need complementary treatment are defined. Many studies have shown that in most cases surgical staging is totally inadequate ${ }^{7.8}$. For example, among the patients operated upon by gynecologists or general surgeons, only $54 \%$ and $35 \%$, respectively, had an appropriate staging procedure, as opposed to $95 \%$ of patients operated upon by gynecologic oncologists. The site most commonly neglected for evaluation was the diaphragm in $83 \%$ of cases $^{7}$. Effective treatment of ovarian cancer includes perfect staging, and for this reason the International Federation of Gynecology and Obstetrics (FIGO) recommends that the extent of disease should be evaluated during surgery as cited above.

For a better understanding of this disease, we conducted a retrospective study of 119 cases seen at the University Hospital of the Ribeirão Preto Medical School, University of São Paulo, from January 1977 to December 1992, with emphasis on staging, general treatment, cytoreduction and second-look operation.

\section{PATIENTS AND METHODS}

A total of 352 patients with ovarian neoplasias were seen at the University Hospital of Ribeirão Preto, University of São Paulo, from January 1977 to December 1992. Of these neoplasias, $119(33.8 \%)$ were malignant and 233 $(66.2 \%)$ were benign. The 119 cases of malignant neoplasias were studied in the present investigation. The following data were obtained from the medical records of the patients: FIGO staging, cytoreduction, general treatment, second-look evaluation, and survival to December 1993.

Cytoreduction was defined according to the following criteria: a) complete when no macroscopic intra-abdominal mass was present after surgery and the cytology of the peritoneal wash or ascitic fluid was negative for malignant cells; b) adequate when one or more masses smaller than 2 $\mathrm{cm}$ were left or the cytology of the peritoneal wash or ascitic fluid was positive for malignant cells; c) inadequate when one or more masses measuring $2 \mathrm{~cm}$ or more were present. Metastatic ovarian cancer was excluded from analysis of stage and cytoreduction.

Statistical analysis of survival time was based on the nonparametric Mann-Whitney test and on the test for two proportions, with the level of significance set at $\mathrm{P}<0.05$.

\section{RESULTS}

Of the 119 cases of malignant neoplasias detected at the University Hospital, Faculty of Medicine of Ribeirão Preto, University of São Paulo, from 1977 to 1992, 70 (58.8\%) were malignant epithelial neoplasias (serous and mucinous adenocarcinoma with 46 and 21 cases, respectively), $21(17.6 \%$ ) were tumors of the sexual girdle/ stroma, $12(10.1 \%)$ were germ cell tumors, $9(7.6 \%)$ were metastatic, $4(3.4 \%)$ were undifferentiated carcinomas, and $3(2.5 \%)$ were of uncertain origin. Tables 1,2 and 3 respectively illustrate the staging, type of treatment used and the evaluation of surgical cytoreduction for all cases of ovarian cancer. From 37 of these patients, staged as IIIB or IIIC, ascitic fluid or peritoneal wash samples were collected during the preoperative period. Cytology was negative for neoplastic cells in 7 (18.9\%) of the cases evaluated.

Iatrogenic rupture of the tumoral capsule occurred in $2(1.8 \%)$ of the 110 cases of original ovarian cancers, one of them being a mucinous tumor (stage IC) and the other a serous tumor (stage IIC). In the specific case of the serous tumor, the postoperative peritoneal wash was negative for neoplastic cells. The patient was submitted to total

Table 1

\begin{tabular}{|c|c|c|}
\hline $\begin{array}{r}\text { Frequ } \\
\text { perf } \\
\text { c }\end{array}$ & $\begin{array}{l}n \text { by } \\
10 \text { ca: } \\
\text { the } \\
\text { om } 1 \text {. }\end{array}$ & $\begin{array}{l}\text { al staging } \\
\text { ovarian } \\
\text { pital, }\end{array}$ \\
\hline Stage & $\mathrm{N}$ & $\%$ \\
\hline 1 & 41 & 37.3 \\
\hline II & 10 & 9.1 \\
\hline III & 55 & 50 \\
\hline IV & 4 & 3.6 \\
\hline Total & 110 & 100 \\
\hline
\end{tabular}


Table 2

Frequency distribution by type of treatment employed for the total number of 119 cases of ovarian cancer treated at the University Hospital, FMRP, from 1977 to 1992.

\begin{tabular}{lcc}
\hline Treatment & N & $\%$ \\
Surgery + Chemotherapy & 59 & 49.6 \\
Surgery & 42 & 35.3 \\
Surgery + Chemotherapy + Radiotherapy & 13 & 10.9 \\
Surgery + Radiotherapy & 5 & 4.2 \\
Total & 119 & 100 \\
\hline
\end{tabular}

Table 3

Frequency distribution by surgical cytoreduction procedure applied to the total number of 110 cases of original ovarian cancer treated at the University Hospital, FMRP, from 1977 to 1992.

\begin{tabular}{lcc} 
Cytoreduction & $\mathrm{N}$ & $\%$ \\
Complete & 43 & 39.1 \\
Adequate & 36 & 32.7 \\
Inadequate & 31 & 28.2 \\
Total & 110 & 100 \\
\hline
\end{tabular}

abdominal hysterectomy (TAH) + bilateral salpingectomy (BSO) + omentectomy (O) and 6 cycles of chlorambucil treatment; the second look (SL) was negative and no signs of recurrence were detected 36 months after treatment.

In the case of the mucinous tumor, cytology was negative for neoplastic cells in ascitic fluid. The patient was submitted to $\mathrm{TAH}+\mathrm{BSO}+\mathrm{O}$ and radiotherapy; SL was negative and the patient was in good general condition 72 months after treatment.

Spontaneous rupture of the tumoral capsule occurred in $3(2.7 \%)$ cases, with staging given in parenthesis: granulosa cells (IC), immature teratoma (IIC) and dysgerminoma (IC). The patient with the granulosa cell tumor was submitted to $\mathrm{TAH}+\mathrm{BSO}+\mathrm{O}+$ chlorambucil (6 cycles); cytology was not performed and SL was negative. After 72 months, the patient was in good general condition. In the immature teratoma case, ascites had neoplastic cells and retrouterine and rectal peritoneal implants were present. This patient was submitted to TAH $+\mathrm{BSO}+\mathrm{O}+$ telecobalt therapy and SL was not performed. A recurrence was detected 11 months after surgery and the patient died within 24 months. In the dysgerminoma case, cytology and SL were not performed. The patient was submitted to $\mathrm{TAH}+\mathrm{BSO}+\mathrm{O}$. Sixty months after surgery she presented no signs of recurrence.

Second-look surgery was performed in 31 (28.2\%) of the 110 cases of non-metastatic ovarian cancer. Twenty $(64.5 \%)$ were positive and $18(90 \%)$ of them were in stage IIIB or IIIC. Of this group of 20 patients, one was lost to follow-up and the survival of the remaining ones ranged from 8 to 72 months after the first surgery $(x=24.2 \pm 15.1$ months). Five were still alive after a follow-up of 20 to 28 months ( $x=22.4 \pm 3.6$ months). On the occasion of the SL, cytoreduction was found to be adequate in $7(35 \%)$ cases, inadequate in $12(60 \%)$ and complete in $1(5 \%)$. Of these 20 patients, five are still alive: three with adequate cytoreduction, two with inadequate and complete cytoreduction, respectively, and one who was lost to follow-up. The mean survival of the seven patients with adequate cytoreduction was $22.4 \pm 12.9$ months and the survival of the 11 patients with inadequate cytoreduction was $25.7 \pm 17.5$ months $(\mathrm{P}=$ not significant $)$.

Comparison of percentage survival between cases of adequate cytoreduction ( 3 patients still alive out of a total of 7) and patients with inadequate cytoreduction (one patient still alive out of a total of 12) using the test for two proportions demonstrated a significant difference $(\mathrm{P}<0.05)$. Of 11 patients with a negative SL, one was lost to follow-up and the remaining ones are still alive after a follow-up of 21 to 156 months $(x=79.4 \pm 48.5$ months $)$.

Statistical analysis by the Mann-Whitney test of survival data after a positive or negative SL demonstrated that, when all the stages are considered together, patients with a negative SL $(n=10)$ had a longer survival than patients with a positive $S L(n=19)$, i.e., $79.4 \pm 48.5$ versus $24.2 \pm 15.1$ months $(\mathrm{P}<0.02)$. Comparison of stages I and II demonstrated that patients with a positive $\mathrm{SL}(\mathrm{n}=3)$ and a negative SL $(n=6)$ had a mean survival of $44.7 \pm 30.4$ and $87.8 \pm 47.7$ months, respectively ( $P=$ not significant $)$. When stages III and IV were compared, patients with a positive SL $(n=16)$ had a mean survival of $19.9 \pm 7.2$ versus $66.8 \pm 54.2$ months for patients with a negative $\mathrm{SL}$ $(n=4)(P<0.02)$.

Analysis of death rate and survival until December 1993 after a positive or negative SL by staging demonstrated that the 6 patients in stages I and II with a negative SL were alive with a mean survival of $87.8 \pm 47.4$ months, whereas the 3 patients with a positive SL died. Of these 3 patients, one showed adequate cytoreduction in the SL and presented a survival of 50 months, and the other two had inadequate cytoreduction and a mean survival of $42 \pm 42.4$ months. The 4 patients in stages III and IV with a negative SL were alive in December 1993, with a mean survival of $66.7 \pm 54.1$ months. Of the 17 patients with a positive SL, $11(68.7 \%)$ died. Of 6 patients with adequate cytoreduction, 3 were alive with a mean survival of $21.3 \pm 2.3$ months, 
and 3 died after a mean survival of $14.3 \pm 3.2$ months. Of 9 patients with inadequate cytoreduction, one was alive with a mean survival of 28 months and 8 died after a mean survival of $32.8 \pm 30.3$ months. One patient with complete cytoreduction was alive, with a survival of 20 months. Comparison of the death rate for adequate and inadequate cytoreduction in stages III and IV with a positive SL showed a significant difference $(\mathrm{P}<0.05$, test for two proportions).

\section{DISCUSSION}

The primary treatment of ovarian cancer should always be surgical ${ }^{3}$. In women who have given birth to all the children they want, surgery should include total hysterectomy with bilateral adnexectomy. Preservation of ovarian hormonal function during the premenopause may be considered, but the benefits do not exceed the risks of preserving the contralateral ovary, which may contain an occult tumor with the risk of subsequent development of cancer.

Among young women who want to have children, if the tumor is in stage I, grade 1 or possibly grade 2 , conservation of the uterus and of the contralateral adnexa is appropriate and widened biopsy and freezing are performed in the preserved ovary. Even though this surgery facilitates the appearance of adhesions that may interfere with conception, techniques of in vitro fertilization may be used. In the cases of stage IA grade 3 or stage IC, surgery is conservative but should be followed by chemotherapy. Few patients have been treated in this way and the risk of recurrence of the disease in the preserved ovary is unknown ${ }^{3}$. In the present study, there were two cases of stage IA which were submitted only to unilateral oophorectomy: in the first case the diagnosis was of well differentiated serous cystadenocarcinoma and the patient (a 71-year old woman) has a survival of 72 months; in the second case, the patient (a 36-year old woman) had a borderline papilliferous mucinous cystadenocarcinoma and a survival of 188 months. Neither patient presents signs of recurrence.

Patients who want to have children should be warned that their children will be at higher risk due to the familial cancer syndrome ${ }^{9}$, since this fact may influence their decision with respect to conservative surgery. Most authors have reported that, after the patients have had the desired number of children, surgery should also be completed due to the risk of developing cancer in the preserved ovary, although this risk has not yet been quantified in the literature.
The second look is defined as reexplorative surgery after a complete chemotherapy schedule in order to detect possible residual disease. This operation is an objective means of determining the response to therapy and has contributed to the development and evaluation of programs of ovarian cancer treatment. The findings obtained by this second surgery are correlated with survival and serve to evaluate the chemotherapeutic and surgical treatment. There are doubts about the individual benefit for patients submitted to a second look, but there are no doubts about the general benefits of treatment of ovarian cancer. The present results demonstrate that survival after a negative SL was three times higher (79.4 versus 24.2 months) compared to a positive SL.

Since the SL is an invasive procedure, some authors have proposed methods of investigation to replace it, especially imaging methods such as ultrasound, computerized tomography and magnetic resonance. However, some studies have demonstrated that these methods are unable to reveal residual lesions smaller than $2 \mathrm{~cm}^{10,11}$. Another method employed is laparoscopy but, due to the number of complications of this procedure in these cases, with the impossibility of resecting large masses and the lack of reliability of negative findings, laparoscopy cannot be considered as a substitute for $\mathrm{SL}^{3}$.

Blood CA-125 measurement has been used to determine the response of the tumor to chemotherapy. However, when used as an indicator of tumor presence at the time of SL, this method is valid only if the level is high, since negativity does not exclude the presence of residual tumors ${ }^{12,13}$.

In the 1960 's, Munnel ${ }^{14}$ introduced the concept termed maximum surgical effect, referring to the increased survival of patients with complete surgery and cytoreduction compared to those with partial surgery. Other studies have demonstrated similar results associated with chemotherapy ${ }^{15-19}$ or radiotherapy ${ }^{20,21}$. Cytoreduction at the time of SL should be considered since approximately $40 \%$ of the patients present clinically identifiable masses. The benefits of this surgery are controversial, but a study conducted at the Sloan-Kettering Memorial Hospital showed that patients with cytoreduction for microscopic disease had a better prognosis than patients who were left with macroscopic disease ${ }^{8}$.

In the present study, the percentage of positive SL was $64.5 \%$. Statistical analysis of the survival of these patients did not show a significant difference between adequate and inadequate cytoreduction. However, the death rate in the groups with adequate cytoreduction was significantly lower $(\mathrm{P}<0.05)$ compared to the group with inadequate cytoreduction. 


\section{CONCLUSIONS}

1. No patient was submitted to biopsy of the diaphragm and material for cytology was not collected from 22 of the 51 tumors in stages I and II ( $43.1 \%)$, procedures that are of great importance for staging.

2. The detection of negative cytology in peritoneal or ascitic fluid during the preoperative period in cases with IIIB and IIIC staging ( 7 cases $=18.9 \%$ of the total of 37 samples) suggests the occurrence of faulty collection, storage or preparation of this material.

3. The patients with a negative second look had a better prognosis compared to those with residual disease. Among the latter, adequate or inadequate cytoreduction on the occasion of SL did not improve prognosis in terms of survival. However, the percentage of patients alive with adequate cytoreduction was significantly higher than the percentage of patients with inadequate cytoreduction.

\section{Resumo}

Objetivos: Este estudo foi conduzido em pacientes com câncer de ovário com o objetivo de avaliar a sobrevida. Desenho: Um estudo retrospectivo de 119 casos de câncer de ovário de janeiro de 1977 a dezembro de 1992 com observação até 1993. Local: Departamento de Ginecologia e Obstetrícia, Faculdade de Nıedicina de Ribeirão Preto, Universidade de São Paulo. Participantes: De 119 casos, 70 (58.8\%) eram adenocarcinomas epiteliais e $21(17.6 \%)$ eram tumores do cordão sexual/ estroma. Fonte de Dados: Os dados foram coletados dos prontuários médicos das pacientes. Mensuração: A análise estatística da sobrevida foi baseada no teste não-paramétrico de Mann-Whitney com significância de $P<0,05$. Resultados: As pacientes com second look negativo tiveram sobrevida de $79,4 \pm 48,5$ meses versus $24,2 \pm 15,1$ meses do second look positivo $(P<0,02)$. Conclusão: Conclui-se que as pacientes com second look negativo apresentam prognóstico melhor em relação àquelas com doença residual.

\section{REFERENCES}

1. Feldman GB \& Knapp RC. Lymphatic drainage of the peritonal cavity and its significance in ovarian cancer. Am J Obstet Gynecol 1974;119:991-4.

2. Piver MS, Barlow JJ, Lele SB. Incidence of subclinical metastasis in stage I and II ovarian carcinoma. Obstet Gynecoly 1978;52:100-4.

3. Rubin SC. Surgery for ovarian cancer. Hematol/Oncol Clin North Amer 1992;6:851-65.

4. Deutsch M, Beck D, Manor D, Brandes J. Metastatic brain tumor following negative second-look operation for ovarian carcinoma. Gynecol Oncol 1987;27:116-9.

5. LeRoux PD, Berger MS, Elliott JP, Tamimi HK. Cerebral metastases from ovarian carcinoma. Cancer 1991;67:21949.

6. Hacker NF, Berek JS, Lagasse LD et al. Primary cytoreductive surgery for epi.helial ovarian cancer. Obstet Gynecol 1983;61:413-20.

7. Dauplat J, Ferriere JP, Gorbinet $\mathrm{M}$ et al. Second-look laparotomy in managing epithelial ovarian carcinoma. Cancer 1986;57:1627-31.

8. Hoskins WJ, Rubin SC, Dulaney E et al. Influence of secondary cytoreduction at the time of second-look laparotomy on the survival of patients with epithelial ovarian cancinoma. Gynecol Oncol 1989;34:365-9.

9. Lynch HT, Bewtra C, Lynch JF. Familial ovarian carcinoma. Clinical nuances. Am J Med 1986;81:1073-6.

10. Lund B, Jacobsen K, Rasch L et al. Correlation of abdominal ultrasound and computed tomography scans with secondor third-look laparotomy in patients with ovarian carcinoma. Gynecol Oncol 1990;37:279-83.

11. Murolo C, Costantini S, Foglia G et al. Ultrasound examination in ovarian cancer patients. A comparison with second look laparotomy. J Ultrasound Med 1989;8:441-5.

12. Berek JS, Knapp RC, Malkasian CD et al. CA 125 serum levels correlated with second-look operations among ovarian cancer patients. Obstet Gynecol 1986;67:685-9.

13. Niloff JM, Bast RJ, Schartzl EM, Knapp RC. Predective value of CA 125 antigen levels in second-look procedures for ovarian cancer. Am J Obstet Gynecol 1985;151:981-6.

14. Munnell $\mathrm{E}$. The changing prognosis and treatment in cancer of the ovary. Am J Obstet Gynecol 1968;100:790-805.

15. Conte P, Sertoli M, Bruzzone M et al. Cisplatin, methotrexate and 5-flourouracil combination chemotherapy for advanced ovarian cancer. Gynecol Oncol 1985;20:290-4.

16. Delgado G, Oram DH, Petrilli ES. Stage III epithelial ovarian cancer: The role of maximal surgical reduction. Gynecol Oncol 1984;18:293-7. 
17. Louie K, Ozols R, Myers E et al. Long term results of a cisplatin-containing combination chemotherapy regimen for the treatment of advanced ovarian carcinoma. J Clin Oncol 1986;4:1579-82.

18. Sutton GP, Stehman FB, Einhorn LH et al. Ten-year followup of patients receiving cisplatin, doxorubicin, and cyclophosphamide chemotherapy for advanced epithelial ovarian carcinoma. J Clin Oncol 1989;7:223-9.
19. Vogl S, Pagano M, Kaplan B et al. Cisplatin based combination chemotherapy for advanced ovarian cancer: High overall response rate with curative potential only in women with small tumor burdens. Cancer 1983;51:2024-30.

20. Dembo A. Radiotherapeutic management of ovarian cancer. Semin Oncol 1984;11:238-41.

21. Fuks Z, Rizel S, Anteby S et al. The multinodal approach to the treatment of Stage IV ovarian carcinoma. Int J Radiat Oncol Biol Phys 1982;8:903-7. 